ACCEPTED MANUSCRIPT

\title{
Observation of Wigner crystal phase and ripplon-limited mobility behavior in monolayer CVD MoS 2 with grain boundary
}

To cite this article before publication: Jyun-Hong Chen et al 2018 Nanotechnology in press https://doi.org/10.1088/1361-6528/aab5ff

\section{Manuscript version: Accepted Manuscript}

Accepted Manuscript is "the version of the article accepted for publication including all changes made as a result of the peer review process, and which may also include the addition to the article by IOP Publishing of a header, an article ID, a cover sheet and/or an 'Accepted Manuscript' watermark, but excluding any other editing, typesetting or other changes made by IOP Publishing and/or its licensors"

This Accepted Manuscript is (c) 2018 IOP Publishing Ltd.

During the embargo period (the 12 month period from the publication of the Version of Record of this article), the Accepted Manuscript is fully protected by copyright and cannot be reused or reposted elsewhere.

As the Version of Record of this article is going to be / has been published on a subscription basis, this Accepted Manuscript is available for reuse under a CC BY-NC-ND 3.0 licence after the 12 month embargo period.

After the embargo period, everyone is permitted to use copy and redistribute this article for non-commercial purposes only, provided that they adhere to all the terms of the licence https://creativecommons.org/licences/by-nc-nd/3.0

Although reasonable endeavours have been taken to obtain all necessary permissions from third parties to include their copyrighted content within this article, their full citation and copyright line may not be present in this Accepted Manuscript version. Before using any content from this article, please refer to the Version of Record on IOPscience once published for full citation and copyright details, as permissions will likely be required. All third party content is fully copyright protected, unless specifically stated otherwise in the figure caption in the Version of Record.

View the article online for updates and enhancements. 


\title{
Observation of Wigner Crystal Phase and Ripplon-Limited Mobility Behavior in Monolayer CVD MoS 2 with Grain Boundary
}

\author{
Jyun-Hong Chen ${ }^{l}$, Yuan-Liang Zhong ${ }^{l, *}$, Lain-Jong Li ${ }^{2}$, and Chii-Dong Chen ${ }^{3}$ \\ ${ }^{1}$ Department of Physics and Center for Nanotechnology, Chung Yuan Christian University, Chung-Li 32023, Taiwan. \\ ${ }^{2}$ Physical Sciences and Engineering Division, King Abdullah University of Science and Technology (KAUST), Huwal 23955- \\ 6900, Kingdom of Saudi Arabia. \\ ${ }^{3}$ Institute of Academia Sinica, Taipei, Taiwan.
}

\begin{abstract}
\end{abstract}
Two-dimensional electron gas (2DEG) is crucial in condensed matter physics and is present on the surface of liquid helium and at the interface of semiconductors. Monolayer $\mathrm{MoS}_{2}$ of 2D materials also contains 2DEG in an atomic layer as field effect transistor (FET) ultrathin channel. In this study, we synthesized double triangular $\mathrm{MoS}_{2}$ through a chemical vapor deposition method to obtain grain boundaries for forming a ripple structure in FET channel. When the temperature was higher than approximately $175 \mathrm{~K}$, the temperature dependence of the electron mobility $\mu$ was consistent with those in previous experiments and theoretical predictions. When the temperature was lower than approximately $175 \mathrm{~K}$, the mobility behavior decreased with the temperature; this finding was also consistent with that of the previous experiments. We are the first research group to explain the decreasing mobility behavior by using the Wigner crystal phase and to discover the temperature independence of ripplon-limited mobility behavior at lower temperatures. Although these mobility behaviors have been studied on the surface of liquid helium through theories and experiments, they have not previously analyzed in 2D materials and semiconductors. We are the first research group to report the similar temperature-dependent mobility behavior of the surface of liquid helium and the monolayer $\mathrm{MoS}_{2}$.

KEYWORDS: Two-dimensional materials, metal transition dichalcogenide, $\mathrm{MoS}_{2}$, field effect transistor, mobility, ripplon, Wigner crystal 


\section{INTRODUCTION}

An electron gas that is free to move in two dimensions, two-dimensional electron gas (2DEG), which is crucial in systems from liquid helium surface to semiconductor interface in condensed matter physics and applied electronic devices. The size of traditional semiconductor transistors has followed Moore's law since 1947, but silicon transistors are reaching their fabrication and physical limits. 2D materials provide the new option of fabricating ultimate thin channel transistors, which can replace silicon transistor in next-generation electronic and optoelectronic devices. ${ }^{1,2} 2 \mathrm{D}$ materials of an atomic layer thickness are also a natural 2DEG system. The first 2D material was discovered in the graphene and its $2 \mathrm{DEG}$ with Dirac energy band structure become a special 2DEG. In traditional $2 \mathrm{DEG}$ of semiconductor such as $\mathrm{SiO} / \mathrm{Si}$ interface and GaAs/AlGaAs heterojunction, electron transport in the idea 2DEG is ballistic transport and the scattering almost happen in channel edge. Semiconductor 2DEG has several crucial scattering mechanisms including the optical and acoustic phonon scattering, electron-electron scattering, piezoelectric scattering, and defect scattering. ${ }^{3}$ Electron mobility is related to the temperature-independent and temperature-dependent scattering time $\tau(T)$ by $\mu=e \tau / m^{*}$, including electron charge $e$ and effective mass $m^{*}$. The mobility behavior increases as temperature decreases at high temperatures because of phonon scattering. ${ }^{4}$ Some scattering mechanisms are different from 2D materials. Phonon scattering in the atomic layer through dielectric environments influences mobility behavior; this phenomenon is discussed in this paper.

Monolayer transition metal dichalcogneides (TMDs) are a group of 2D materials. The $\mathrm{MoS}_{2}$ TMDs has a direct band gap $(1.8 \mathrm{eV})$ similar to that of semiconductors..$^{5}$ The valley band structure of $\mathrm{MoS}_{2}$ is similar to that of graphene with a hexagonal lattice structure and Dirac energy band structure. However, $\mathrm{MoS}_{2}$ has a nonzero band gap, an inversion symmetric lattice and strong spin-orbit coupling, which distinguishes it from graphene. ${ }^{6}$ Its properties make it suitable for the development of next-generation transistors and novel valleytronics. ${ }^{1,6}$ Field effect transistor (FET) with ultrathin channel made from monolayer and bulk $\mathrm{MoS}_{2}$ have been reported in some studies. The bulk MoS 2 electron mobility is in the range of $200-500 \mathrm{~cm}^{2} \mathrm{~V}^{-1} \mathrm{~s}^{-1}$ at nearly room temperature ${ }^{7,8}$ and the exfoliation of monolayer on $\mathrm{SiO}_{2}$ substrate was found to result a mobility of only $0.1-10 \mathrm{~cm}^{2} \mathrm{~V}^{-1} \mathrm{~s}^{-1} \cdot 9$ A dielectric engineering technique was proposed for improving carrier mobility in low-dimensional semiconductor by modifying the dielectric environments. ${ }^{10}$ In a recent experiment, a high-k gate dielectric comprising a $\mathrm{HfO}_{2}$ layer in a top-gated FET has an enhance a mobility of approximately $200 \mathrm{~cm}^{2} \mathrm{~V}^{-}$ ${ }^{1} \mathrm{~s}^{-1} .{ }^{11}$ Theoretical results revealed Coulomb impurities can be strongly suppressed by dielectric environments and that the intrinsic phonon-limited mobility can be increased to approximately $410 \mathrm{~cm}^{2} \mathrm{~V}^{-1} \mathrm{~s}^{-1} \cdot{ }^{12}$ At high-temperature, the temperature dependence of mobility is expected to follow $\mu \sim T^{\gamma}$, where $\gamma=1.69$; the coefficient can be reduced to 1.52 by quenching 
of the homopolar mode in dielectric environments. ${ }^{12}$ While the monolayer chemical vapor deposition $(\mathrm{CVD}) \mathrm{MoS}_{2}$ exhibits defects from grain boundary, the mobility is likely limited by scattering from grain boundary defects. ${ }^{13}$ Grain boundaries were observed as a characteristic of the space-charge-limited current, and the current-voltage characteristics of one switching cycle demonstrated memristive behavior. ${ }^{14}$ Grain boundaries form a ripple structure in 2D materials. ${ }^{15-17}$

Ripples in 2D material lead a different 2DEG behavior compared with semiconductor heterostructures, but the similar 2DEG behavior also exist on the surface of liquid helium. Previous studies obtained the behavior of electron mobility on the surface of liquid helium divided into three temperature regions: the helium vapor atom scattering region, the Wigner crystal phase, and ripplon-limited mobility. In this study, we synthesized double-triangular MoS 2 using CVD method to obtain grain boundaries for forming ripple structure in monolayer $\mathrm{MoS}_{2}$. We also observed a Wigner crystal phase and the electron gas became an electron crystal because of electron localization in the insulating state at a temperature of less than approximately $175 \mathrm{~K}$.

Electron mobility was also discovered to be limited at less than $150 \mathrm{~K}$. This ripplon-limited mobility was limited by the scattering of electron by ripplons in the 2DEG. ${ }^{18,19}$ Ripplons are quantized capillary waves (ripples) caused by surface tension. On the other hand, we designed a dual-gate configuration to investigate the effect of dielectric environment. Our results yielded the coefficient $\gamma=1.62$ for the side gate and $\gamma=1.73$ for the back gate, which are close to the theoretical value of 1.69 at temperatures ranging from $300 \mathrm{~K}$ to approximately $175 \mathrm{~K}$ and consisted with previous experiment. We first found the similar temperature-dependent mobilities behavior between the surface of liquid helium and the monolayer $\mathrm{MoS}_{2}$ because of a ripple membrane-like 2 DEG in the both systems.

\section{EXPERIMENT METHOD}

We synthesized high-quality monolayer single-crystal $\mathrm{MoS}_{2}$ on a sapphire substrate by using a CVD method. Our experimental setup is schematically illustrated in Figure $1 \mathrm{a} . \mathrm{MoO}_{3}(0.4 \mathrm{~g})$ and sulfur $(0.8 \mathrm{~g})$ powders were placed in two ceramic boats, and a sapphire substrate was placed on the top of a boat. To synthesize $\mathrm{MoS}_{2}$, the reaction chamber was heated to $150^{\circ} \mathrm{C}$ in a nitrogen and argon environment for an annealing time of $20 \mathrm{~min}$, after which the temperature was increased to $650^{\circ} \mathrm{C}$ and maintained for 20 min. Finally, $\mathrm{MoO}_{3}$ diffused onto the sapphire substrate and reacted with sulfur vapor to synthesize $\mathrm{MoS}_{2}$. Figure $1 \mathrm{~b}$ presents an optical image of $\mathrm{MoS}_{2}$ and reveals single, double and mirror-double triangles on the sapphire substrate. 


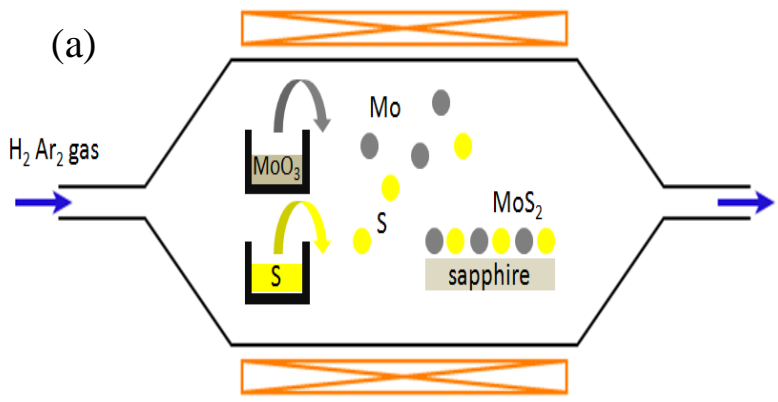

(b)

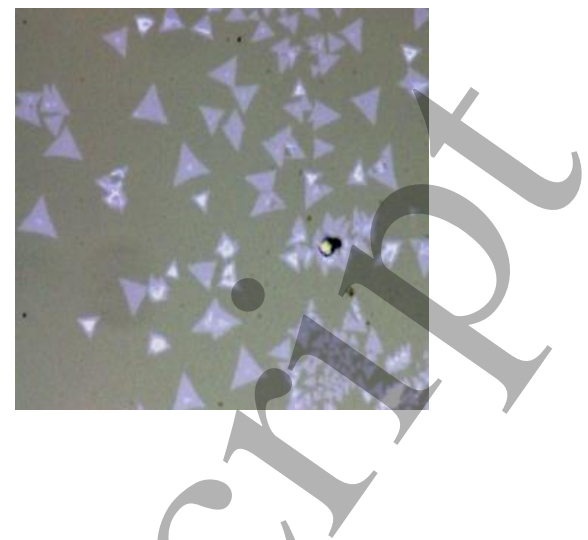

Figure 1. (a) Schematic of the synthesis process of CVD MoS 2 . The $\mathrm{MoS}_{2}$ film was synthesized on sapphire through the evaporation of Mo (gray) and sulfur (yellow) in nitrogen and argon gas flow. (b) Optical images of monolayer $\mathrm{MoS}_{2}$ triangle on the sapphire substrate.

We transferred the $\mathrm{MoS}_{2}$ triangle crystal on to a transmission electron microscopy (TEM) grid, measured their crystal structure, and analyzed the Mo and S compounds through field emission TEM (JEOL JEM-2100). Figure 2a displays a TEM high-resolution TEM image of single-crystal $\mathrm{MoS}_{2}$, and the atomic percentage ratio of Mo to $\mathrm{S}$ was found to 1:2 by analyzing the TEM-based energy dispersive spectroscopy spectrum (TEM-EDS) presented in Figure $2 \mathrm{~b}$. The imagines and analysis demonstrate the high-quality structure of our monolayer $\mathrm{MoS}_{2}$ triangles.

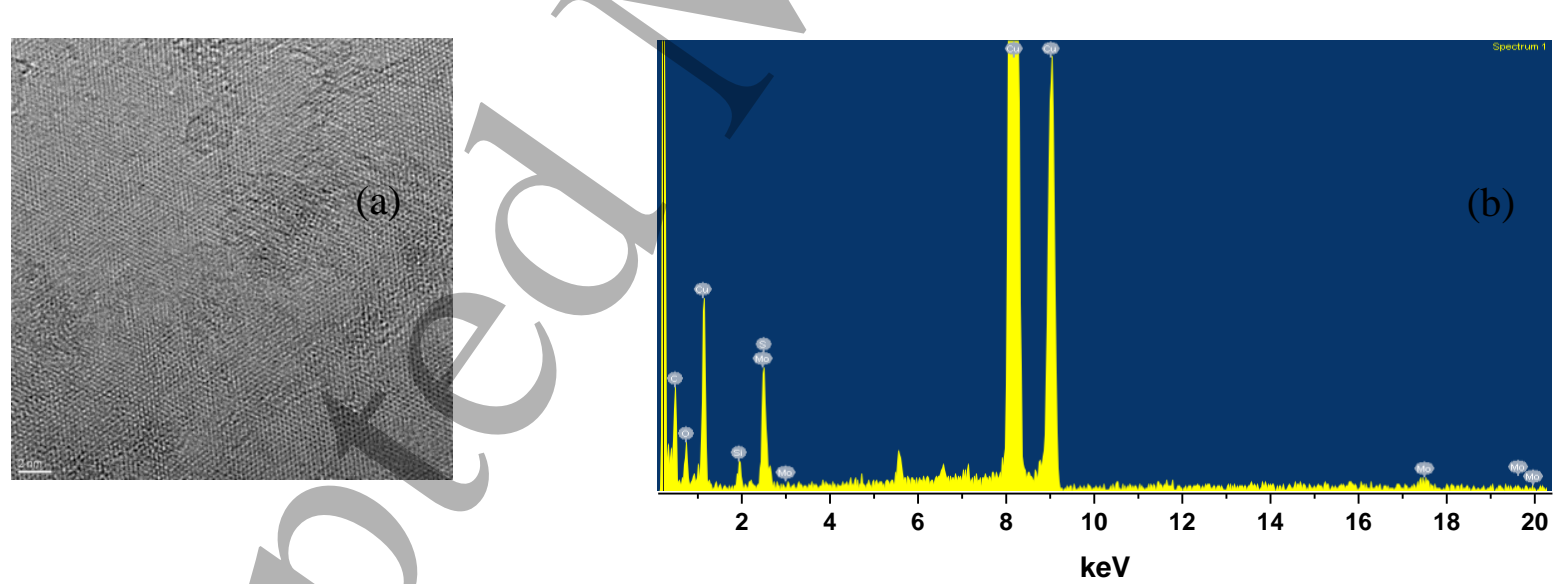

Figure 2. (a) TEM images of $\mathrm{MoS}_{2}$ film; scale bar represents $2 \mathrm{~nm}$. (b) TEM-EDS spectrum used to analyze the Mo:S ratio.

We transferred the monolayer $\mathrm{MoS}_{2}$ from the sapphire to a $\mathrm{SiO}_{2}$ substrate by using $\mathrm{NaOH}$ solution to etch sapphire. The PMMA/MoS 2 films was floated upon $\mathrm{NaOH}$ solution for $1 \mathrm{~h}$, and then transferred to deionized water for dilution. Finally, monolayer $\mathrm{MoS}_{2}$ was transferred onto a $\mathrm{SiO}_{2}$ (300-nm thickness)/Si substrate upon which electrodes had already been patterned. Figure $3 \mathrm{a}$ shows monolayer $\mathrm{MoS}_{2}$ with double triangle and the metallic Ni (20 nm)/Au (50 nm) electrodes 
that were fabricated on the triangular sample through electron-beam lithography. We designed a monolayer MoS $\mathrm{FET}_{2}$ and Figure 3c shows that the transistor's structures had a double tringle in between source and drain electrodes and two gates, one gate of which was on the channel beside and the other was on the bottom of the substrate. The grain boundaries in channel area are between the source and drain electrodes (Figure 3b).
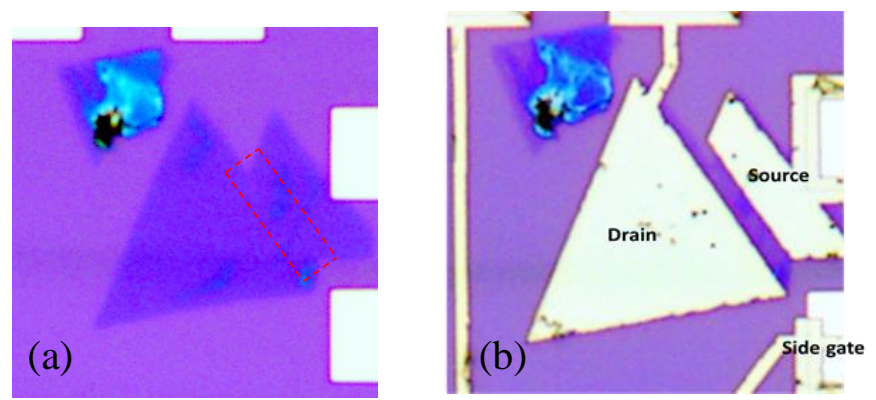

(c)

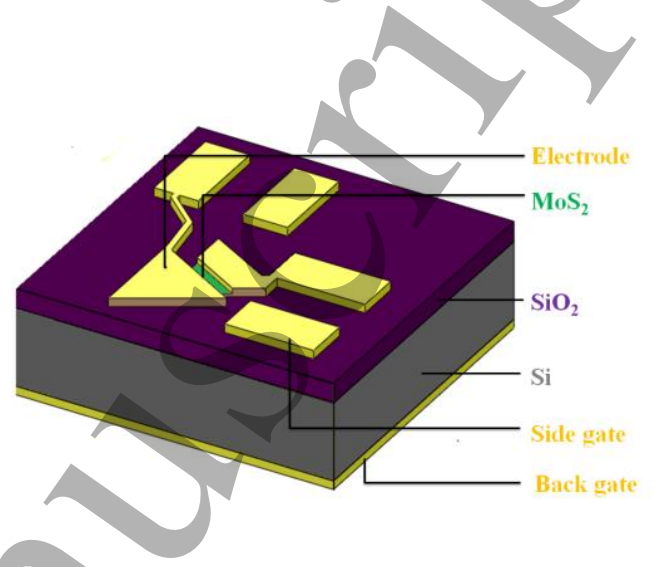

Figure 3. (a) Optical image of monolayer $\mathrm{MoS}_{2}$, including double triangle on the $\mathrm{SiO}_{2} / \mathrm{Si}$ substrate (b) Optical image of $\mathrm{MoS}_{2}$ FET. The metallic Ni /Au electrodes fabricated on double triangles let the grain boundary between two electrodes (source and drain). (c) A schematic of the $\mathrm{MoS}_{2}$ FET with two electrodes, a gate beside the two electrodes, and another gate on the bottom of the chip.

\section{RESULTS AND DISCUSSION}

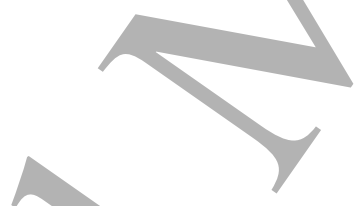

We first characterized the channel of the double-triangular $\mathrm{MoS}_{2}$ in the monolayer $\mathrm{MoS}_{2}$ FET by applying a bias voltage $V_{\mathrm{ds}}$ between the source and drain electrodes and a voltage $V_{\mathrm{g}}$ on the back gate, which created an out-of-plane electric field, or on the side gate, which created an in-plane electric field. The characteristics of electron transport in our MoS 2 FET were thus determined using the back-gate voltage $V_{\mathrm{gb}}$ or side-gate voltage $V_{\mathrm{gs}}$ to control the current $I_{\mathrm{ds}}$ between the source and drain at room temperature or for varying temperature down to liquid helium temperature. Figure 4 displays the electrical characteristics of the $\mathrm{MoS}_{2}$ FET when $V_{\mathrm{gb}}$ was applied at room temperature. 

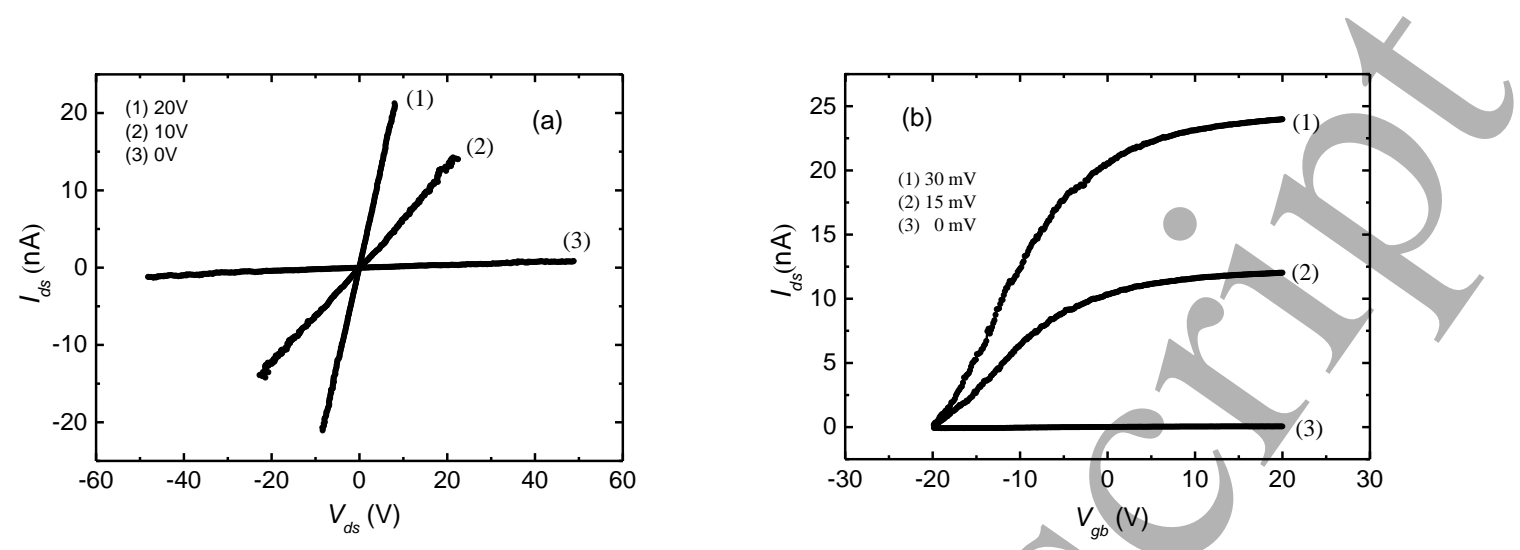

Figure 4. Electrical characterization of monolayer $\mathrm{MoS}_{2}$ FET when $V_{\mathrm{gb}}$ was applied at the room temperature; (a) $I_{\mathrm{ds}}-$ $V_{\mathrm{ds}}$ curves for $V_{\mathrm{gb}}=0,10$ and $20 \mathrm{~V}$. (b) $I_{\mathrm{ds}}-V_{\mathrm{gb}}$ curves for a bias voltage $V_{\mathrm{ds}}$ with a range of approximately 10 to $30 \mathrm{mV}$ and a side gate bias $\mathrm{V}_{\mathrm{gs}}$ with a range of -20 to $20 \mathrm{~V}$.

Figure $4 \mathrm{a}$ reveals the linear dependence of the current on the bias voltage $\left(I_{\mathrm{ds}}-V_{\mathrm{ds}}\right)$, indicating device electrodes with Ohmic contacts. $I_{\mathrm{ds}}$ was controlled through typical back-gating and revealed the $V_{\mathrm{gb}}$ dependence of $I_{\mathrm{ds}}\left(I_{\mathrm{ds}}-V_{\mathrm{gb}}\right.$ curves), indicating an $n$-channel depletion FET (Figure 4b). Furthermore, we obtained similar electrical characteristics for the same device when $V_{\mathrm{gs}}$ was applied. 
1

2

3

4

5

6

7

8

9

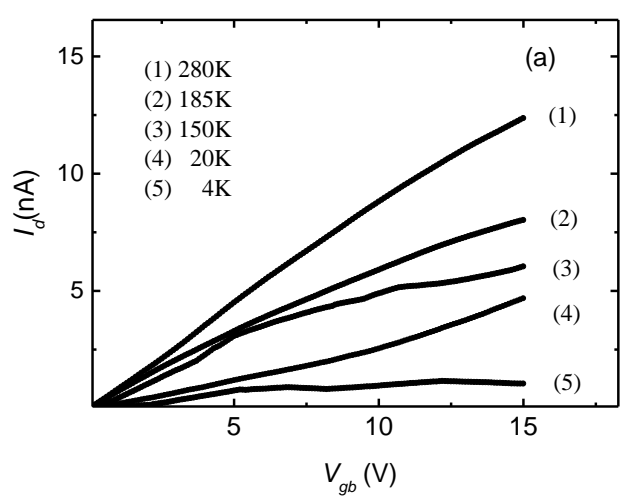

Figure 5. Temperature dependence of gating current and mobility; (a) normalized $I_{\mathrm{ds}}-V_{\mathrm{gb}}$ curves obtained for $T=280,185,150$, 20 and $4 \mathrm{~K}$. (b) The mobility $\mu$ as a function of temperature when a voltage was applied to the side gate (open circles) and back gate (close triangles). At the temperature above around $190 \mathrm{~K}$ (side gate) or $165 \mathrm{~K}$ (back gate), $\mu$ is limited by phonon scattering and has a $T^{-\gamma}$ dependence, where $\gamma=1.73$ (dashed line; for side gate) or $\gamma=1.62$ (solid line; for back gate), as obtained by fitting the experimental data. Between approximately 200 and $150 \mathrm{~K}$, the solid and dashed lines are trend lines to guide the eye. At less than approximately $150 \mathrm{~K}$, the mobility approaches a temperature independence that indicates ripplon-limited mobility behavior. This mobility temperature dependence behavior is similar to that of surface-state electrons on liquid helium.

Figure 5a presents the $I_{\mathrm{ds}}-V_{\mathrm{gb}}$ curves obtained at various temperatures. We extracted the field effect mobility $\mu$ from the slope of $I_{\mathrm{ds}}-V_{\mathrm{gb}}$ curves by using the expression $\mu=\left[L /\left(W C_{\mathrm{ox}} V_{\mathrm{d}}\right) \times\left(d I_{\mathrm{ds}} / d V_{\mathrm{g}}\right)\right],{ }^{20}$ where the length $L$ and width $W$ of the $\mathrm{MoS}_{2}$ channel was $10 \mu \mathrm{m}$ and $l \mu \mathrm{m}$, respectively. $C_{\mathrm{ox}}=\epsilon_{0} \epsilon_{\mathrm{r}} / d$ was the electric capacity per area, where $\epsilon_{0}$ is the vacuum dielectric constant and $\epsilon_{\mathrm{r}}$ is relative dielectric constant of $\mathrm{SiO}_{2}$ layer (dielectric thickness $d$ ) between $\mathrm{MoS}_{2}$ and silicon. The values of $\epsilon_{\mathrm{r}}$ and $d$ are $3.9^{21}$ and $300 \mathrm{~nm}$, respectively, for obtaining $C_{\mathrm{ox}}=1.15 \times 10^{-4} \mathrm{~F} \mathrm{~m}^{-2}$. We subsequently determined the temperature dependence of the mobility $\mu$, which is illustrated in Figure 5b. The mobility as a function of temperature $T$ show the mobility behavior with a peak at around $190 \mathrm{~K}$ (side gate) and $165 \mathrm{~K}$ (back gate) and then down to the values of temperature independence below around $150 \mathrm{~K}$. In our observation, mobility has three temperature-dependent regions: the phonon-limited (electron-phonon interaction) region at higher temperatures, the Wigner crystal (electron-electron interaction) region at medium temperatures and the ripplon-limited (electron-boundary or defect interaction) region at lower temperature. The mobility of electrons in monolayer $\mathrm{MoS}_{2}$ has generic temperature dependence represented by $\mu \sim T^{-\nu}$, where the exponent $\gamma$ depends on the dominant scattering mechanism in the different temperature ranges. In theories, the mobility mechanism originates at high temperature (approximately $\mathrm{T}>100 \mathrm{~K}$ ) from the physics of electron scattering by intrinsic optical phonon, which has the dependence $\mu \sim T^{-1.69}$, and at medium or low temperature (approximately $T<100$ 
$\mathrm{K})$, scattering by intrinsic acoustic phonons, which has the dependence $\mu \sim T^{-1}$. Electron mobility is also limited by dielectric environment effects caused by elastic scattering from located Coulomb impurities at substrate surface, and surface optical phonons. ${ }^{12,22-24}$ In our results, the temperature dependence of mobility follows $\mu \sim T^{\gamma}$ with $\gamma=1.62$ and 1.73 when voltage was applied to the side and back gate, respectively, of the $\mathrm{MoS}_{2}$ FET at temperatures exceeding $165 \mathrm{~K}$. The theoretical prediction is $\gamma=1.69$, which was calculated using a model of the intrinsic phonon-limited mobility via dielectric engineering. ${ }^{12}$ We fabricated the bake gate from dielectric $\mathrm{SiO}_{2}$, and the side gate was empty dielectrics for the dielectric engineering. Previous experiments and theories have indicated the strong suppression of charged impurity scattering for gates in dielectric environment. ${ }^{10,12,25,26}$ Our results did not reveal a clearly different behavior, but a temperature shift was identified between the mobility obtained when voltage was applied to the bake and side gates (Figure 5b).

In the temperature dependence curves, the mobility changed abruptly at approximately 190 and $165 \mathrm{~K}$ for side-and back-gate voltage applications, respectively. Such abrupt mobility changes have also been observed in previous experiments, but no theory has yet been proposed to explain the behavior. ${ }^{10,27}$ We consider that the abrupt decrease with decreasing temperature indicated the Wigner crystal phase, after which the mobility becomes temperature-independent plateau (Ripplon-limited mobility behavior) because of the scattering of electron by ripplons at low temperature. The ripplon-limited mobility of surface electron on liquid helium has previously been reported, ${ }^{18,19,28}$ but it has not been observed in the $2 \mathrm{DEG}$ of semiconductors and 2D materials. 2D materials with grain boundaries can form ripple structures in the ultrathin atomic layer. 2DEG in 2D materials can be considered similar with that electrons on the surface of liquid helium. We designed the FET device with a grain boundary in the channel between two electrodes to study ripplon-limited mobility.
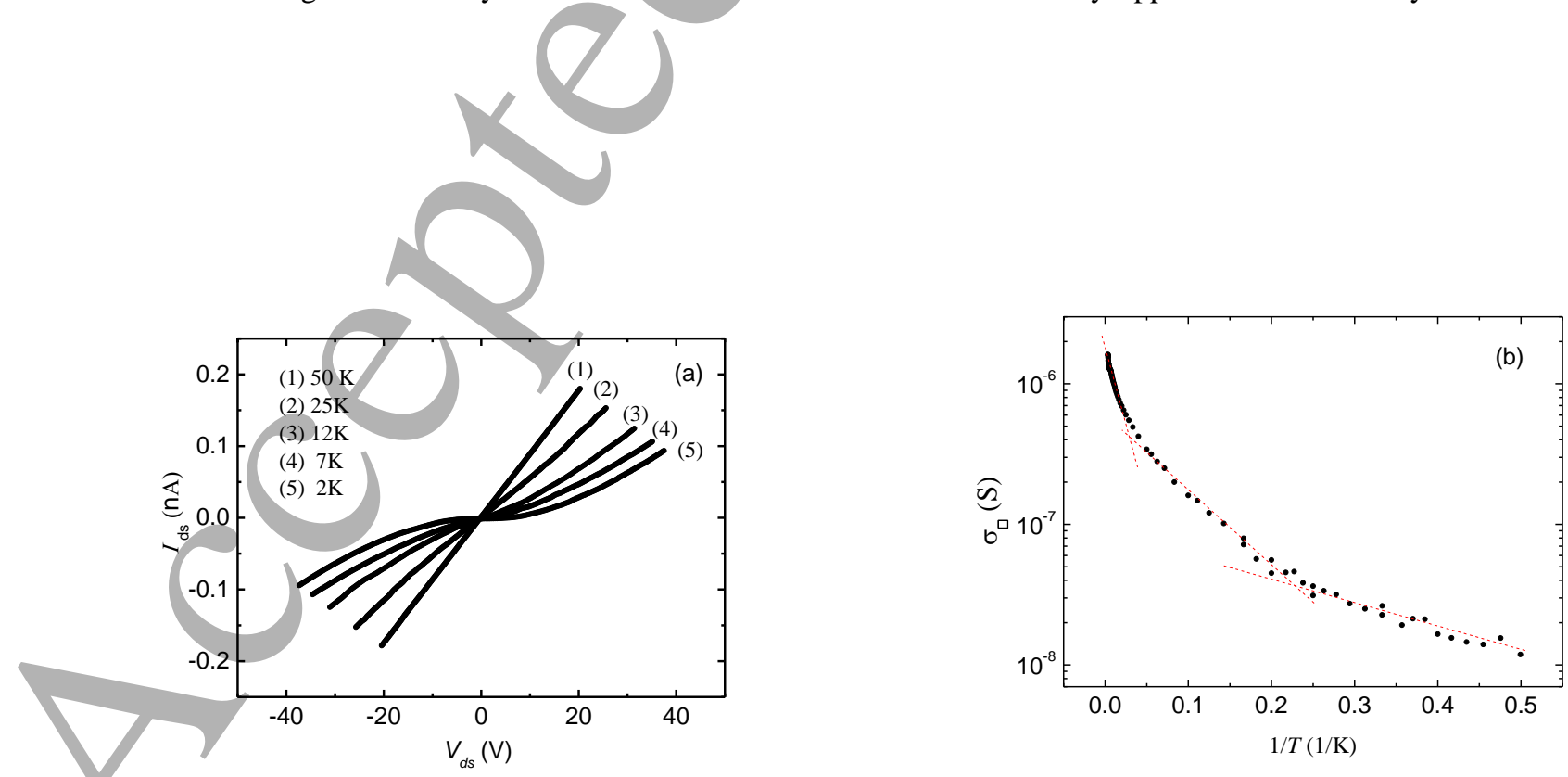
Figure $6 \mathrm{a}$ show the $I_{\mathrm{ds}}-V_{\mathrm{ds}}$ curves obtained at various temperature, which become nonlinear as the temperature is decreased. The $I_{\mathrm{ds}}-V_{\mathrm{ds}}$ curves indicate a change in conduction from metallic behavior to insulating behavior when the temperature is decreased. The sheet conductivity $\sigma_{\square}$ as function of temperature also indicates a metal-insulator transition behavior (Figure 6b). This transition was also reported in previous experiments on MoS $_{2}$ FET. ${ }^{10}$ The insulating behavior has been interpreted as being caused by Wigner crystal phase. ${ }^{25,}{ }^{29}$ Electron move by hopping between localized electron states. Typically, electron hopping transport occurs through thermal phonon with a thermally activated energy $E_{h}$ at relatively high temperatures, as indicated in eq. 1 , where $T_{0}=E_{h} / k_{B}$ and $n=1$. The conduction mechanism at medium temperatures, Mott hopping transport dominates though the phonon to assist electron tunneling process. The terms $n$ and $T_{0}$ are $1 / 3$ (in two dimensions) and $T_{M}$, respectively, in eq. (1). At relatively low temperatures, the terms $n$ and $T_{0}$ are $1 / 2$ and $T_{E S}$, respectively, in eq. 1, and Efros-Shklovskii hopping transport dominates the hopping processes, with an enhanced electron-electron interaction. The conductivity of hopping mechanisms follows the temperature dependence of conductivity, which is given by 26,30

$$
\sigma(T)=\sigma_{0} \exp \left[-\left(T_{0} / T\right)^{\mathrm{n}}\right]
$$

Figure $6 \mathrm{~b}$ shows a qualitative guide to the eye of three dashed lines with different slopes, which correspond to three hopping mechanisms. An electron propagates in monolayer $\mathrm{MoS}_{2}$ as a Bloch wave showing a metallic transport that can be changed to a charge trap as a localized state showing an insulating transport. The charge trap effect induces a metal-insulator transition when the temperature decreased, electron transport becomes dominated by variable range hopping. ${ }^{10,22}$ The metal-insulator transition, with the insulating behavior being observed at lower temperatures and carrier densities, has been interpreted to be caused by Wigner crystal phase. ${ }^{29,31}$ Electrons in the $2 \mathrm{DEG}$ in monolayer $\mathrm{MoS}_{2}$ become localized to form $2 \mathrm{D}$ electron lattice (Wigner crystal). The electron mobility behavior of Wigner crystal has also been observed in surface electron on liquid helium. In a heterostructure semiconductor system, Wigner crystal was observed in very pure samples and in a strong magnetic field. ${ }^{32}$ The Wigner crystal phase is the first to be observed in liquid helium in a zero magnetic field and in this study is the first to be 
observed in solid state systems in a zero magnetic field. In 2D materials, although graphene is expected to exhibit Wigner crystal phase in theory ${ }^{33}$, it is not demonstrated in experiment so far. The $\mathrm{MoS}_{2}$ with a lower electron density is superior to graphene for demonstrating Wigner crystal phase in experiments. In our experiments, we have observed that the temperature dependence electron mobility in monolayer $\mathrm{MoS}_{2}$ was similar with that of surface electron on liquid helium.

Wigner first calculated an electron-liquid to electron-solid phase transition in the 3D Fermi system at low densities in 1934. A classical Coulomb system determine the thermodynamic state by measuring the ratio of Coulomb potential energy $U_{\mathrm{c}}$ to kinetic energy $K_{\mathrm{e}}$. In the classical $2 \mathrm{D}$ electron system the ratio is $\Gamma=U_{\mathrm{c}} / K_{\mathrm{e}}=e^{2}(\pi n)^{1 / 2} / k_{\mathrm{B}} T_{M} \cdot 32,34$. When $\Gamma \gtrsim 100$, the Coulomb potential energy dominates and then the electrons are expected to because a periodic classical 2D electron crystal (classical Wigner crystal). The melting transition is characterized by the parameter $P^{32,34}$ We estimate the melting temperature $T_{\mathrm{M}}$ of $\mathrm{MoS}_{2}$ by $\Gamma$ comparing with the surface of liquid helium. In the surface of liquid helium system, the $T_{\mathrm{M}}$ was measured to obtain $0.457 \mathrm{~K}$ and the $\Gamma$ was estimated to obtain 137 by electron density $n=4.4 \times 10^{8}$ in previous experiment. ${ }^{34}$ If $\Gamma=137$, we estimated the $T_{\mathrm{M}}$ of $\mathrm{MoS}_{2}$ by $n=5 \times 10^{12}$ of $\mathrm{MoS}_{2}$, the $T_{\mathrm{M}}$ was obtained approximately $50 \mathrm{~K}$, and if $\Gamma=100$, the $T_{\mathrm{M}}$ was obtained approximately $70 \mathrm{~K}$. Although the value of $T_{\mathrm{M}}$ is lower than our measuring value of approximately $175 \mathrm{~K}$, this value is higher than liquid helium over two orders of magnitude. The estimating values were obtained by using formula $\Gamma$ that is not included dielectric effect in the classical theory. Our estimating results indicate that the higher $T_{\mathrm{M}}$ is possible to happen in $2 \mathrm{D}$ materials. We may need a new theory including a special $2 \mathrm{D}$ band structure and quantum effect to predict the value of $T_{\mathrm{M}}$, and for higher electron density.

\section{CONCLUTION}

We synthesized double triangular $\mathrm{MoS}_{2}$ crystal using CVD method to obtain grain boundaries and a dual-gate configuration was designed with back and side gate for studying the effect of a dielectric environment. The doubletriangular sample synthesis and good contact in electrodes are difficult to obtain simultaneously. The temperature dependence of the electron mobility was measured in the monolayer $\operatorname{MoS}_{2}$ FET and discovered to follow $\mu \sim \mathrm{T}^{-\gamma}$ with $\gamma=$ 1.62 and 1.73 when the different electric field directions was applied through voltage on the side and back gates for temperature higher than approximately 190 and $165 \mathrm{~K}$, respectively. This compared favorably with the theoretical prediction of $\gamma=1.69$. The temperature dependence of the electron mobility was consistent with those in previous experiments and theoretical predictions. The mobility behavior decreased with the temperature; this finding has also been observed in previous experiments. We are the first research group to explain the decreasing mobility behavior through the Wigner crystal 
phase and to discover the temperature independence of ripplon-limited mobility behavior at a lower temperature. Although these mobilities behavior have been studied on the surface of liquid helium through theories and experiments, they have not previously been analyzed in 2D materials and semiconductors. We are the first research group to report the similar temperature-dependent mobility behavior of the surface of liquid helium and the monolayer MoS $\mathrm{M}_{2}$ Although we were difficult to obtain more simples, the temperature dependence of the electron mobility behavior was consistent with those of previous experiments and agreed with the value of theoretical prediction at a higher temperature range. The temperature dependence of the electron mobility behavior was also consistent in liquid helium 2DEG. Our results provide a suggestion for theorists to calculate electron mobility at medium and low temperatures and a new 2DEG material system for studying 2D Wigner crystal in details in future experiments. The future experiments are necessary and interesting for $2 \mathrm{D}$ Wigner crystal in 2D materials to study in details.

\section{AUTHOR INFORMATION}

Corresponding Author:

* E-mail: ylzhong@cycu.edu.tw (Yuan-Liang Zhong)

\section{CONFLICT OF INTEREST}

The authors have no completing financial interest to declare.

\section{ACKNOWLEDGMENT}

This study was funded by the National Science Council of Taiwan (MOST 104-2112-M-033-006).

\section{REFERENCES}

1. Wang, Q. H.; Kalantar-Zadeh, K.; Kis, A.; Coleman, J. N.; Strano, M. S. Nat Nanotechnol 2012, 7, (11), 699-712. 
2. Fiori, G.; Bonaccorso, F.; Iannaccone, G.; Palacios, T.; Neumaier, D.; Seabaugh, A.; Banerjee, S. K.; Colombo, L. Nat Nanotechnol 2014, 9, (10), 768-779.

3. Walukiewicz, W.; Ruda, H. E.; Lagowski, J.; Gatos, H. C. Phys Rev B 1984, 30, (8), 4571-4582.

4. $\quad$ Pfeiffer, L.; West, K. W.; Stormer, H. L.; Baldwin, K. W. Appl Phys Lett 1989, 55, (18), 1888-1890.

5. Mak, K. F.; Lee, C.; Hone, J.; Shan, J.; Heinz, T. F. Phys Rev Lett 2010, 105, (13), 136805.

6. Xiao, D.; Liu, G. B.; Feng, W.; Xu, X.; Yao, W. Phys Rev Lett 2012, 108, (19), 196802.

7. Fivaz, R.; Mooser, E. Physical Review 1967, 163, (3), 743-755.

8. $\quad$ Bao, W.; Cai, X.; Kim, D.; Sridhara, K.; Fuhrer, M. S. Appl Phys Lett 2013, 102, (4), 042104.

9. Novoselov, K. S.; Jiang, D.; Schedin, F.; Booth, T. J.; Khotkevich, V. V.; Morozov, S. V.; Geim, A. K. P Natl Acad Sci USA 2005, 102, (30), 10451-10453.

10. Radisavljevic, B.; Kis, A. Nat Mater 2013, 12, (9), 815-20.

11. Radisavljevic, B.; Radenovic, A.; Brivio, J.; Giacometti, V.; Kis, A. Nat Nanotechnol 2011, 6, (3), 147-50.

12. Kaasbjerg, K.; Thygesen, K. S.; Jacobsen, K. W. Phys Rev B 2012, 85, (11).

13. van der Zande, A. M.; Huang, P. Y.; Chenet, D. A.; Berkelbach, T, C.; You, Y. M.; Lee, G. H.; Heinz, T. F.; Reichman, D. R.; Muller, D. A.; Hone, J. C. Nat Mater 2013, 12, (6), 554-561.

14. Sangwan, V. K.; Jariwala, D.; Kim, I. S.; Chen, K. S.; Marks, T. J.; Lauhon, L. J.; Hersam, M. C. Nat Nanotechnol 2015, 10, (5), 403-406.

15. Miro, P.; Ghorbani-Asl, M.; Heine, T. Adv Mater 2013, 25, (38), 5473-5.

16. Capasso, A.; Placidi, E.; Zhan, H. F.; Perfetto, E.; Bell, J. M.; Gu, Y. T.; Motta, N. Carbon 2014, 68, $330-336$.

17. Luo, S.; Hao, G.; Fan, Y.; Kou, L.; He, C.; Qi, X.; Tang, C.; Li, J.; Huang, K.; Zhong, J. Nanotechnology 2015, $26,(10), 105705$.

18. Shirahama, K.; Ito, S.; Suto, H.; Kono, K. Journal of Low Temperature Physics 1995, 101, (3), 439-444.

19. Monarkha, I. U. P.; Kono, K., Two-dimensional Coulomb liquids and solids. Springer: Berlin ; New York, 2004 ; p xi, 346 p.

20. Fuhrer, M. S.; Hone, J. Nat Nano 2013, 8, (3), 146-147.

21. Streetman, B. G.; Banerjee, S., Solid state electronic devices. Seventh edition. ed.; Pearson: Boston, $2015 ;$ p xviii, 596 pages.

22. Yu, Z.; Ong, Z.-Y.; Li, S.; Xu, J.-B.; Zhang, G.; Zhang, Y.-W.; Shi, Y.; Wang, X. Adv Funct Mater 2017, $27,(19), 1604093$.

23. Yu, Z.; Ong, Z. Y.; Pan, Y.; Cui, Y.; Xin, R.; Shi, Y.; Wang, B.; Wu, Y.; Chen, T.; Zhang, Y. W.; Zhang, G.; Wang, X. Adv Mater 2016, 28, (3), 547-52.

24. Ong, Z.-Y.; Fischetti, M. V. Phys Rev B 2013, 88, (16).

25. Yoon, J.; Li, C. C.; Shahar, D.; Tsui, D. C.; Shayegan, M. Phys Rev Lett 1999, 82, (8), 1744-1747.

26. Pollak, M.; Shklovski1̌, B. I., Hopping transport in solids. New York, NY, USA, 1991; p xiv, 453 p.

27. Zhu, W.; Low, T.; Lee, Y. H.; Wang, H.; Farmer, D. B.; Kong, J.; Xia, F.; Avouris, P. Nat Commun $2014,5,3087$.

28. Grimes, C. C.; Adams, G. Phys Rev Lett 1976, 36, (3), 145-148.

29. Varró, S.; Sólyom, J.; Ádám, P.; Biró, T. S.; Barnaföldi, G. G.; Lévai, P. EPJ Web of Conferences 2014, 78, 01009. 
30. Gantmakher, V. F., Electrons and disorder in solids. New York, 2005; p x, 225 p.

31. Abrahams, E.; Kravchenko, S. V.; Sarachik, M. P. Reviews of Modern Physics 2001, 73, (2), 251-266.

32. Monarkha, Y. P.; Syvokon, V. E. Low Temp Phys 2012, 38, (12), 1067-1095.

33. Dahal, H. P.; Joglekar, Y. N.; Bedell, K. S.; Balatsky, A. V. Phys Rev B 2006, 74, (23).

34. Grimes, C. C.; Adams, G. Phys Rev Lett 1979, 42, (12), 795-798.

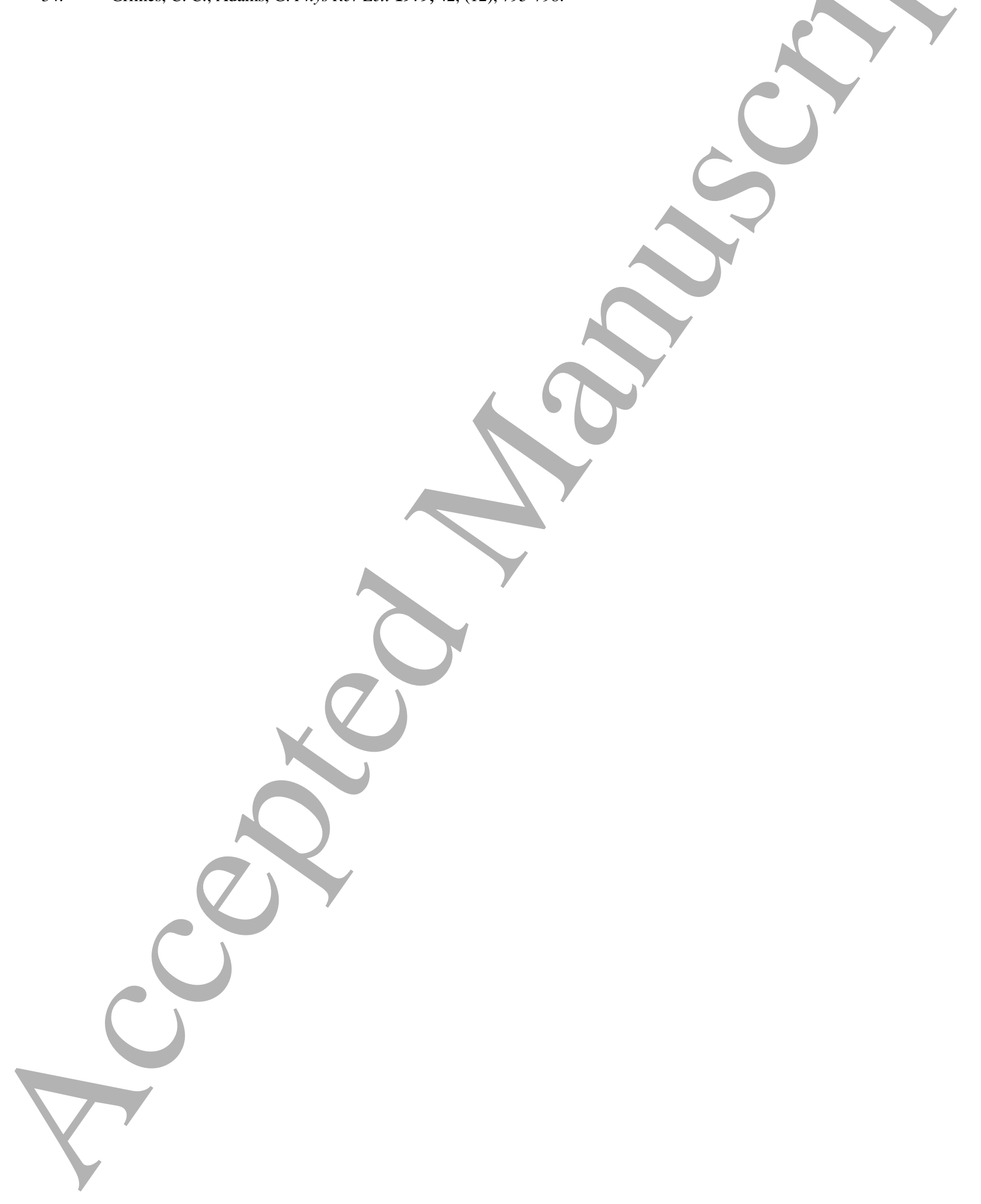

